\title{
Crime and Safety among Women Travellers in Kuala Lumpur City
}

\author{
Rohan Sham¹, Muhammad Zaly Shah Muhamad Hussein², \\ Hairul Nizam Ismail2 \\ ${ }^{1}$ Faculty of Business and Information Science, UCSI University Kuala Lumpur, Malaysia \\ 2 Faculty of Built Environment, Universiti Teknologi Malaysia, Malaysia \\ rohana@ucsiuniversity.edu.my
}

\begin{abstract}
Fighting with a high level of fear every day while travelling to work, had put the desire for them to switch from public to private transport usage if they have a choice. Main aim of the study is to determine the interaction of a few urban design factors and transport service characteristics to further analyze on the issue of safety of women travellers. A quantitative method were considered, where a passengers bus survey is use as a form of data collection. This study contributes to improve the current urban design and service provision policy in creating a safer environment for many women commuters.
\end{abstract}

Keywords: Crime;Travel safety; Vulnerable commuters; Urban environment

eISSN 2398-4295 @ 2018. The Authors. Published for AMER ABRA cE-Bs by e-International Publishing House, Ltd., UK. This is an open-access article under the CC BY-NC-ND license (http://creativecommons.org/licenses/bync-nd/4.0/). Peer-review under responsibility of AMER (Association of Malaysian Environment-Behaviour Researchers), ABRA (Association of Behavioural Researchers on Asians) and CE-Bs (Centre for EnvironmentBehaviour Studies), Faculty of Architecture, Planning \& Surveying, Universiti Teknologi MARA, Malaysia.

http://dx.doi.org/10.21834/ajbes.v3i11.99 


\subsection{Introduction}

Many women travellers who stay in an urban area show the highest risk of victimization. These had affected on their level of fear while travelling especially for primary journey. Working women who lives in an urban area who depend on public transport like buses face higher level of fear due to many unknown factors. Rising in the participation of women in a working sector, had also contributed to a criticality of the issue because they were left with no option to travel to work purpose.

\subsection{Literature Review}

When travelling is concern, it involves several travel component in which infrastructure is one of the important component that travellers had to deal with in order to complete a journey. Providing a practical infrastructure does not benefit a group of women travellers only, but it will also create lots of continuous benefit towards the rest of the society as the provision of the infrastructure would be used by all users. A large and growing body of literature has investigated the effect of the environment design provision towards the feeling of safety among the travellers.

A recent study done by Siti Rashidah et al in 2013 had focus on the gated and non gated residential area and feeling of fear among the resident. However, their study did not focus on the bus commuters and their travelling pattern among women who live and had to travel from the gated and non gated area into the city centre for working purpose.

\subsection{Urban Environment design}

Having known that women travelling pattern differ in so many ways as compared to men, their needs must first be address in enhancing their level of safety while travelling. A practical infrastructure design was discussed in this paper in line with the needs of these commuters to travel safe especially when working trip in urban areas are concern. Since women are considered as the most vulnerable group especially towards the crime occurrence, an urgent attention is needed especially on the allocation, and design of the infrastructure to aid their travelling activities.

A large and growing body of literature has investigated the effect of the infrastructure provision towards the travellers feeling of safe. These studies often highlighted the qualities of the infrastructure Apart from highlighting the quality issues of the infrastructure design provision, the previous literature has also discussed the vital contribution of the infrastructure design towards the criminal behaviour or crime incident. Numerous studies have attempted to explain that poor provision of the infrastructure would increase the risk of criminal activity.

The growing of literature on infrastructure provision started to be discussed critically in 1980s. Morris (1980). further identified that both men and women, young and older people felt most unsafe after dark waiting or walking to and from the bus stop. However, he pointed out that the majority of the respondent felt secure while being on the bus as compared to the waiting environment or when walking to and from the stop. Hence, the study concluded that the quality of the traveling environment had been highlighted by bus users as an crucial factor influencing fear of crime. Poor maintenance of public infrastructure such as graffiti, vandalism 
and litter along the way to and from the stop and at the station itself signaled to users that the place was in poor supervision and lack of control in which it created high feeling of fear. To further look into the matter, Benjamin (1994) investigated the differential impact of infrastructure provision and maintenance in Worcestershire.

According to Prendergrast (2006), many avoided the station whenever they could, especially outside off peak travelling times and at night. Although there was little actual crime, the station was frequently dark and deserted, which added to people's fear. However after the surrounding the transport services, he also revealed that isolated bus stops and unstaffed station deterred potential public transport users and increased use of private car. One of the successful case studies in Liverpool bus station area indicated that the station was a place where people felt unsafe before. According to Prendergrast (2006), many avoided the station whenever they could, especially outside off peak travelling times and at night. Although there was little actual crime, the station was frequently dark and $d$ station had been turned around by an upgrading of the station which include improved lighting, replacement of low-growth shrubs with a single stem trees to improve sight lines, implementation of regularly monitored close circuit television and opening of restaurant and retail shop nearby had significantly reduced the feeling of fear among the bus passengers especially women and thus increased the number of people using the station. Prendergrast (2006) also revealed that there has been a $14 \%$ increase in the number of bus passenger in the morning and $17 \%$ increase in the afternoon peak. But even better was the massive $77 \%$ increase use after $6 \mathrm{pm}$. While it was being acknowledged that the increase was because of the longer working hour in the city centre, but it was still believed that the increase was because people felt much safer. The study concluded that the qualities of the infrastructure design that had been highlighted by public transport users as important factors influencing fear of crime.

\subsection{Crime prevention through environmental design}

It is believed that through this theory, crime could be prevented using an appropriate environmental design. Therefore analyzing the guideline of a practical environmental design is deem as influential as level of fear could be reduce through a specific design.

\subsection{A practical environmental design policy}

According to US Department of Transport (2001) sustainable development is viewed as development that improves service quality, the standard of living, and quality of life, while at the same time protecting and enhancing the natural environment and honouring local culture and history. To be able to develop a sustainable infrastructure design, several reputable practises were being outlined by the Department of Transport (2006). Among the proper practice being highlighted in the guideline were as follows:

\subsubsection{Bus stop design}

- Making the bus stop clearly visible, to avoid passengers missing their stop 
- Naming stops and shelters with locally recognizable names, to reduce confusion and the potential for conflict between passenger and driver, and to promote a sense in which the service is part of the local community

- Ensuring that the stop or shelter is well-lit, or located in an area that is generally well-lit

- Shelters designed to provide maximum visibility and with toughened glass to resist vandalism

- Locating a public telephone close to the bus stop

\subsubsection{Walkways design}

Good practice as indicated by the Department for Transport (2006) includes:

- Maximize sitting and design of the buses especially on the aisle

- The internal design of the bus to maximize clear sight lines and visibility for both driver and passengers

- The use of CCTV surveillance to deter vandalism and other misuse, especially on double deck buses

- Radio contact for the driver to summon help if required

- On local bus services, passenger security can be enhance by the use of a regular bus driver known to frequent travellers

- Presence of revenue inspectors enhances their perception of personal security for on-bus travel especially for service during off peak

- Greater uniform on bus presence through protocol and by providing free travel to police and traffic warden.

\subsection{Service provision policy}

There are a few discussion on the transport service characteristics that create a dilemma between the women travellers.

\subsubsection{Punctuality of the bus service}

A considerable amount of literature has been published on the punctuality of the bus and level of safety. However not all literature supported that the punctuality of the bus affected women level of safety. In 1972, Pagano and Robert reported that there were several bus service quality that would affect the women's level of safety. In 1979, Wigan and Morris who conducted a study of an urban area in Australia had pointed out that a major constraint on the location of $A$ and $B$ was measured by the time and travel between them. They carried out several investigations and found that the common factor for all travellers was that each has only 24 hours to devote to a day's activities. 
In 2001, Transport and Travel Research carried out a number of investigations to analyse critically the attributes of punctuality pertaining to the safety matters. From the survey, it was found that most women claimed that to keep waiting time to a minimum is the most important because this is one of the parts of the whole journey where they felt most insecure.

In 2002, a study by Crime Concern had further reviewed the literature from the early period and found that lack of punctuality of the bus services especially during the evening off peak hours or at the weekend, could contribute to feeling of insecurity, especially if the information on the services was not available or reliable. However, the research did not take into account the feeling of safety if the bus was not punctual at a different location, which possessed different level of infrastructure and different level of crime rate.

\subsubsection{Reliability of the bus service}

Mark (1981) agreed that the concept of service reliability had come into increasing prominent in recent years as an important characteristic of the transport service provisions, but it was still less significant as compared to the concept of security on the transportation service when safety issues were a concern. Another study by Hamilton and Jenkins (1989) in West Yorkshire supported the statement by measuring both component and revealed that when women were to ask to determine the factor that would enhance their level of safety while travelling, they had rated proper waiting places as the most salient attribute and reliability of the bus service as the least influential factors to them. Furthermore, they added that the design and lighting of waiting place were therefore particularly pertinent to ensure a safe ride on a public bus. In other words, they labelled the comfortability of the bus as a subject of critical issues that need further attention whenever safety issues were being discussed.

\subsubsection{Security of the services}

During the past 30 years much more information has become available on the security aspect of services and the level of safety. Security has been identified as a considerable contributional factor for the high level of fear or feeling of unsafe indicated by most of the urban bus commuters (Crime concern, 2002). Early study done by Pagano and Robert (1972) have identified eight general categories or aspect of quality as it relates to the transit industry. The quality being identified are reliability and on time performance, comfort, convenient, extent of service, vehicle access, safety, driver characteristic and responsiveness. From all the characteristics mentioned, he pointed out that safety and security, and on time performance (punctuality) were found to be the most notable aspect from the user's point of view especially when women's travel safety was concern.

\subsection{Methodology}

On the question of the design of the urban environment for women travelers had affected women level of safety, the study found that those who indicated a higher dissatisfaction level towards the infrastructure provision in their area, tend to rate a higher level of unsafe while travelling on the whole journey. Data collection was collected through a pilot survey in Kuala Lumpur Metropolitan Region. As depicted in Table 1, the result indicated that most of the 
current infrastructure design was rated as dissatisfied by the daily women bus commuters. What was appealing in this data was that the most dissatisfaction towards the infrastructure provision was on the street lighting and bus stop lighting. This findings have grave implications for developing a sustainable transport infrastructure provision where allocation of ample lighting to brighten up the walking area and waiting area should be given a top priority in enhancing the level of safety among the bus commuter.

Table 1: Mean score for current infrastructure provision

\begin{tabular}{ll}
\hline Infrastructure & Stage Bus Mean Score \\
\hline Street Lighting & 1.66 \\
Pedestrian Pavement & 2.64 \\
Fence & 2.22 \\
Bus Stop Visibility & 2.09 \\
Bus Stop Location & 3.62 \\
Bus Stop Lighting & 1.57 \\
Walkways to Work Place & 2.13 \\
\hline
\end{tabular}

*1-Very Dissatisfied; 7-Very Satisfied

To further understand the link between the level of satisfaction and the level of safety indication while travelling, analysis on the mean score for level of safety indication while travelling on the whole journey were being carried out. Table 2 provides the overall feeling of safe for stage bus users through the breakdown of four main travelling component namely 'walking, 'waiting', while being in the vehicle' and while walking to work place'.

Table 2: Mean Score for Level of Safety indication While Travelling On The Whole Journey

\begin{tabular}{ll}
\hline Travel Component & Stage Bus Mean Score \\
\hline Walking & 2.18 \\
Waiting & 2.98 \\
In Vehicle & 3.89 \\
Walking To Work Place & 3.47 \\
\hline
\end{tabular}

*1-Very unsafe; 7-Very Safe

It is apparent from the table that most stage bus users indicated that they felt higher feeling of unsafe while walking and waiting. The level of safety while in the vehicle was much higher as compared to while walking to work place. The present result had successfully identified that those who indicated a higher level of dissatisfaction towards the street lighting and bus stop lighting had indicated a higher level of unsafe while walking and waiting at the bus stop. An implication of this result is the possibility that women who indicated a higher level of dissatisfaction towards the infrastructure provision tend to show a higher level of unsafe while travelling. Therefore, in order to develop a successful and sustainable infrastructure design, the need of the women commuters has to be taken into consideration in every aspect of the planning. 


\subsection{Conclusion}

It is generally accepted that adequate, efficient and most importantly a safe infrastructure provision or in this study known as urban travelling environment are essential for most urban dwellers especially women commuters. As developing countries like Malaysia strive to increase their level of infrastructure provision, safety issues seldom being put into top priority that had constitutes towards a higher feeling of unsafe and fear of crime to most women. The main contribution of this research is the analysis of the level of safety indication given a poor or dissatisfied infrastructure provision to aid the travelling activities. The result shows that to be able to achieve a practical infrastructure design, the planning and design of any infrastructure provision should that take into consideration the needs and the involvement of women's travelling activities. Moreover, the maintenance of the provision of any public transport service or infrastructure should be carried out from time to time in order to create the feeling that the areas are well monitored. This is perhaps a hugely important aspect of all planning decision as a well maintains area would create a safer feeling not only to women travellers but also to the entire disadvantage group.

\section{Acknowledgement}

Many thanks go to family and friends for the completion of this paper. Utmost thanks to Universiti Teknologi MARA Cawangan Segamat for resources and Ministry of Higher Education for funding of the study under the Research Acculturation Grant (600-RMI/RAGS $5 / 3$ (131/2012)provided. My heartfelt thanks also go to lecturer of Business Management from UiTM Malaysia and UTM Skudai Johor for their support in completion of this paper.

\section{References}

Benjamin, J. M. (1994). Perception and incidence of crime on public transit in small system in the Southeast. Transportation Journal, 1433, 195-200.

Morris, J. M. (1980). Perception and evaluation of the journey to work. Transportation Journal, 4(2), 20-27.

Prendergrast, M. (2006). Plan it safe: From wasteland to heartland. Liverpool: Crime Prevention Division.

Department for Transport (2000). Why personal security? Retrieved April 2004 from http://www.dft.gov.uk.

Department for Transport (2006). Get on Board: An Agenda for Improving Personal Security- Guidance.

Pagano, S. \& Robert, T. (1972). Who notice, who cares? Passengers reaction to transit safety measures. Transportation Journal, 2, 131-142.

Transport and Travel Research (2001). Perception of safety from crime on public transport: Department Of Transport.

Mark, E. (1981). Environmental consideration in transport project assessment: The need for additional research. Australia: Australian Road Research.

Hamilton, K. \& Jenkins, L. (1989). Why women and travel. Transportation Journal, 23, 18-55. 